\title{
A Control Reduced Primal Interior Point Method for a Class of Control Constrained Optimal Control Problems*
}

\author{
Martin Weiser \\ Tobias Gänzler \\ Anton Schiela
}

June 3, 2008

\begin{abstract}
A primal interior point method for control constrained optimal control problems with PDE constraints is considered. Pointwise elimination of the control leads to a homotopy in the remaining state and dual variables, which is addressed by a short step pathfollowing method. The algorithm is applied to the continuous, infinite dimensional problem, where discretization is performed only in the innermost loop when solving linear equations. The a priori elimination of the least regular control permits to obtain the required accuracy with comparatively coarse meshes. Convergence of the method and discretization errors are studied, and the method is illustrated at two numerical examples.
\end{abstract}

AMS MSC 2000: 90C51, 65N15, 49M15

Keywords: interior point methods in function space, optimal control, finite elements, discretization error

\section{Introduction}

Optimal control problems with PDE constraints are not only very important in practical applications, but also very expensive to solve numerically. Independently of where discretization is located in an algorithm, at the outermost loop as in direct methods or at the innermost loop as in function space oriented methods, the resulting finite dimensional equations are quite large. This is particularly true if highly local features of the solution need to be represented accurately, which requires fine meshes and thus leads to large finite dimensional subproblems.

In control constrained optimization problems such local features arise at the boundaries of active sets, where the control exhibits kinks or even jumps in

*Supported by the DFG Research Center MATHEON "Mathematics for key technologies" in Berlin. The original publication is available at www.springerlink.com (DOI 10.1007/s10589-0079088-y, http://www.springerlink.com/content/t0n5827m2634lq16/?p=e598839a7e094eaf9bb37c35bd364212\&pi=0). 
bang-bang control problems. Unfortunately, the boundaries of active sets are in general not grid-aligned. This leads to error estimates of $\mathcal{O}(h)$ for piecewise constant and $\mathcal{O}\left(h^{3 / 2}\right)$ for piecewise linear control discretizations [1, 4, 15]. For the faithful representation of an approximate solution up to a requested accuracy, massive mesh refinement along the boundaries of active sets is necessary - see e.g. [22] and Fig. 5. In certain situations, the need for refinement can be alleviated by a special postprocessing procedure [12], which results in an approximation order of $\mathcal{O}\left(h^{2}\right)$.

As a different approach to alleviate the need for mesh refinement, Hinze [10] suggests to eliminate the control $u$ analytically from the optimality system by computing it from the dual variable $\lambda$. For a certain class of optimal control problems, this is a simple pointwise calculation. The resulting variables that have to be discretized, the state $y$ and dual variable $\lambda$, are comparatively smooth across boundaries of active sets. For this reason, a rather coarse mesh is sufficient, and a control error of order $\mathcal{O}\left(h^{2}\right)$ is obtained. The approach leads to a nonsmooth equation that has to be solved. For this purpose, semismooth Newton methods are an appropriate choice. Local superlinear convergence has been shown, e.g., in $[11,18,9]$.

A popular alternative to semismooth Newton methods are interior point methods, which substitute the nonsmooth problem by a homotopy of smooth ones. Both primal and primal-dual adaptive function space oriented linearly convergent interior-point methods for optimal control problems have been developed, analyzed and applied in [21, 20, 22, 14]. Superlinear convergence has been obtained recently in [19] by means of an intermediate smoothing step closely related to the current setting.

These methods rely on approximating the control in a finite dimensional space and hence need to refine the mesh substantially, which impedes their computational efficiency. The abovementioned idea of eliminating the control can also be applied to primal interior point methods, which is addressed in this paper. Compared to the interior point methods above, the necessity of mesh refinement is decreased to a similar amount as reported in [10]. A further advantage is the superlinear convergence, which is shown in the companion article [17].

The remainder of the paper is organized as follows: The elimination of the control from the optimality system and its interior point regularization is described in Section 2, where also convergence of the central path is stated. Section 3 is devoted to linear convergence of a short step pathfollowing method, whereas discretization error estimates are given in Section 4. Finally, numerical examples illustrate the method in Section 5. 


\section{Elimination of controls}

For ease of presentation we restrict the discussion to the simple elliptic optimal control problem

$$
\min _{y \in H_{0}^{1}, u \in L^{2}} \frac{1}{2}\left\|y-y_{d}\right\|_{L^{2}}^{2}+\frac{\alpha}{2}\|u\|_{L^{2}}^{2} \quad \text { subject to } \quad L y=u, \quad-1 \leq u \leq 1
$$

on some domain $\Omega \subset \mathbb{R}^{d}, d \leq 3 . y_{d} \in L^{2}$ is the desired state, and $\alpha>0$ is a fixed regularization parameter. $L y=-\operatorname{div}(a(x) \nabla y)+b(x) y$ with symmetric $a(x) \in \mathbb{R}^{d \times d}$ uniformly positive definite and $b(x) \in \mathbb{R}$ uniformly positive is a second order elliptic differential operator. With $S: H^{-1} \rightarrow H_{0}^{1}$ we denote the symmetric positive definite solution operator for the state equation.

Since $H^{3}$-regularity is needed in $\S 4$ in order to derive discretization error estimates for quadratic finite elements, we assume $a \in C^{1,1}(\bar{\Omega}), b \in C^{0,1}(\bar{\Omega})$, and $\partial \Omega \in C^{3}$.

We do emphasize, however, that the following discretization concept can be directly extended to more complex and nonlinear control constrained problems as long as (i) the control constraints are defined pointwise and (ii) the derivative of the objective function w.r.t. the control is an invertible Nemyckii operator of $u$.

For problem (1) the first order necessary conditions state the existence of Lagrange multipliers $\lambda \in H_{0}^{1}$ and $\underline{\eta}, \bar{\eta} \in L^{2}$, such that

$$
\begin{aligned}
y-y_{d}+L \lambda & =0 \\
\alpha u-\lambda-\underline{\eta}+\bar{\eta} & =0 \\
L y-u & =0 \\
\langle\underline{\eta}, u+1\rangle=\langle\bar{\eta}, 1-u\rangle & =0 \\
u+1,1-u, \underline{\eta}, \bar{\eta} & \geq 0 .
\end{aligned}
$$

On the one hand, it is now possible to use (2), (4), and (5) in order to eliminate

$$
u=u(\lambda)=\max (-1, \min (\lambda / \alpha, 1)),
$$

which results in the nonsmooth system

$$
\begin{array}{r}
y-y_{d}+L \lambda=0 \\
L y-u(\lambda)=0 .
\end{array}
$$

This well known formulation is used by [10] to construct a discretization scheme, where only $y$ and $\lambda$ are actually discretized and $u$ is pointwisely computed from $\lambda$.

For later use we notice that the nonsmooth system may be reformulated equivalently as

$$
u=u\left(S\left(y_{d}-S u\right)\right)
$$

where the iteration variable $u$ can be discretized implicitly in terms of discrete approximate solutions of $S\left(y_{d}-S u\right)$. 
On the other hand, primal-dual interior point methods substitute (4) by the regularized equations

$$
\begin{aligned}
\underline{\eta}(u+1)=\bar{\eta}(1-u) & =\mu \quad \text { a.e. } \\
u+1,1-u & >0
\end{aligned}
$$

for $\mu>0$ and thus define the central path $\mu \mapsto(y, u, \lambda, \eta, \bar{\eta})$. This approach has been analyzed and justified in $[20,22]$. Using (7) to eliminate the Lagrange multipliers $\underline{\eta}$ and $\bar{\eta}$ results in the primal interior point method given by

$$
\begin{aligned}
y-y_{d}+L \lambda & =0 \\
\alpha u-\lambda-\frac{\mu}{u+1}+\frac{\mu}{1-u} & =0 \\
L y-u & =0 \\
u+1,1-u & >0 .
\end{aligned}
$$

These are just the first order necessary conditions for the logarithmic barrier reformulation of (1),

$\min \frac{1}{2}\left\|y-y_{d}\right\|_{L^{2}}^{2}+\frac{\alpha}{2}\|u\|_{L^{2}}^{2}+\mu\|\log (u+1)+\log (1-u)\|_{L^{1}} \quad$ subject to $\quad L y=u$.

Existence and convergence of the central path defined by primal interior point methods for control constrained problems has been established in [14], along with convergence of a function space oriented pathfollowing method.

Again, we can use (8) and (9) in order to eliminate $u=u(\lambda)$. Instead of the $L^{2}$-projection (6) we need to solve a scalar cubic equation in every point in space. As before, we obtain the equation system

$$
\begin{aligned}
y-y_{d}+L \lambda & =0 \\
L y-u(\lambda ; \mu) & =0,
\end{aligned}
$$

which is, however, smooth. Its unique solvability is a consequence of the following Lemma.

Lemma 2.1. For any $\mu>0, \lambda \in \mathbb{R}$, there is exactly one $u(\lambda ; \mu) \in]-1,1[$ that satisfies (8). Moreover, $u(\lambda ; \mu)$ is twice continuously differentiable with respect to $\lambda$, and

$$
0 \leq u^{\prime}(\lambda ; \mu) \leq \frac{1}{\alpha+2 \mu} \quad \text { and } \quad\left|u^{\prime \prime}(\lambda ; \mu)\right| \leq \min \left(\frac{1}{\sqrt{\mu \alpha^{3}}}, \frac{8}{\mu^{2}}\right) .
$$

Proof. The left hand side of (8) as a function of $u \in]-1,1[$ is continuous, monotonically increasing and tends to $\pm \infty$ for $u \rightarrow \pm 1$. Therefore, by the intermediate value theorem, equation (8) has a root $u(\lambda)$ for each $\lambda \in \mathbb{R}$. By strict monotonicity this root is unique. 
As for the derivatives, we apply the implicit function theorem to (8) and obtain

$$
\left(\alpha+\frac{\mu}{(u(\lambda ; \mu)+1)^{2}}+\frac{\mu}{(1-u(\lambda ; \mu))^{2}}\right) u^{\prime}(\lambda ; \mu)-1=0,
$$

which gives the first order bound. A second application of the implicit function theorem gives

$$
\begin{aligned}
\left(-\frac{\mu}{(u(\lambda ; \mu)+1)^{3}}+\right. & \left.\frac{\mu}{(1-u(\lambda ; \mu))^{3}}\right) u^{\prime}(\lambda ; \mu)^{2} \\
& +\left(\alpha+\frac{\mu}{(u(\lambda ; \mu)+1)^{2}}+\frac{\mu}{(1-u(\lambda ; \mu))^{2}}\right) u^{\prime \prime}(\lambda ; \mu)=0
\end{aligned}
$$

which yields

$$
u^{\prime \prime}(\lambda ; \mu)=\mu \frac{(u(\lambda ; \mu)+1)^{-3}-(1-u(\lambda ; \mu))^{-3}}{\left(\alpha+\frac{\mu}{(u(\lambda ; \mu)+1)^{2}}+\frac{\mu}{(1-u(\lambda ; \mu))^{2}}\right)^{3}} .
$$

For the case $u(\lambda ; \mu) \leq 0$ we infer

$$
\begin{aligned}
\left|u^{\prime \prime}(\lambda ; \mu)\right| & \leq \mu \frac{(u(\lambda ; \mu)+1)^{-3}}{\left(\alpha+\frac{\mu}{(u(\lambda ; \mu)+1)^{2}}+\frac{\mu}{(1-u(\lambda ; \mu))^{2}}\right)^{3}} \\
& \leq \mu\left(\alpha(u(\lambda ; \mu)+1)+\frac{\mu}{u(\lambda ; \mu)+1}\right)^{-3} \\
& \leq \frac{\mu}{\max (2 \sqrt{\alpha \mu}, \mu / 2)^{3}}=\min \left(\frac{1}{\sqrt{\mu \alpha^{3}}}, \frac{8}{\mu^{2}}\right) .
\end{aligned}
$$

Due to symmetry, this bound is also valid for $u(\lambda ; \mu)>0$.

Remark 2.2. Special care has to be taken for the numerically stable evaluation of $u(\lambda)$. A naive implementation may lead to numerical instabilities for certain ranges of $\alpha, \lambda$, and $\mu$.

We define $v=(y, \lambda)$ and the homotopy

$$
F(v ; \mu)=\left[\begin{array}{c}
y-y_{d}+L \lambda \\
L y-u(\lambda ; \mu)
\end{array}\right]=0,
$$

which in turn defines the central path $v(\mu)$.

Theorem 2.3. For each $\mu>0$ there is a corresponding unique $v(\mu) \in H_{0}^{1} \times H_{0}^{1}$ satisfying (10). $v(\mu)$ is a continuously differentiable path with $\left\|v^{\prime}(\mu)\right\|_{H^{1}} \leq \frac{c}{\sqrt{\mu}}$ for some generic constant $c$ independent of $\mu$. Moreover, the estimate

$$
\|v(\mu)-v(\sigma \mu)\|_{H^{1}} \leq c(1-\sqrt{\sigma}) \sqrt{\mu}
$$

holds. In particular, $v(\mu)$ converges to the solution $v(0)$ of (1) at a rate of

$$
\|v(\mu)-v(0)\|_{H^{1}} \leq c \sqrt{\mu} \text {. }
$$


Proof. This result is a direct consequence of [14]. Alternatively, existence of central path solutions for $\mu>0$ can be shown directly by applying Schauder's fixed point theorem to $u=u\left(S\left(S u-y_{d}\right)\right)$. Continuous differentiability of the path results from the implicit function theorem. The necessary invertibility of $\partial_{v} F(v ; \mu)$ is shown in Lemma 3.3. Integrating the slope of the central path over the interval $[\sigma \mu, \mu]$ yields the estimate (11). Setting $\sigma=0$ we obtain (12).

\section{A pathfollowing method}

This section is devoted to the analysis of a pathfollowing method for solving (10), which employs an inexact Newton corrector. The exact Newton correction $\Delta v^{k}$ defined by the Newton equation

$$
\partial_{v} F\left(v^{k} ; \mu^{k}\right) \Delta v^{k}=-F\left(v^{k} ; \mu^{k}\right)
$$

is numerically unavailable due to discretization and iteration errors. Thus, we resort to inexact Newton methods, where an inner residual $r^{k}$ remains when computing the numerically available inexact Newton correction $\delta v^{k}$ by

$$
\partial_{v} F\left(v^{k} ; \mu^{k}\right) \delta v^{k}=-F\left(v^{k} ; \mu^{k}\right)+r^{k} .
$$

Algorithm 3.1.

select $\mu^{0}>0, \delta>0,0<\sigma<1$, and $v^{0}$ with $\left\|v^{0}-v\left(\mu^{0}\right)\right\|_{H^{1}} \leq \rho \sqrt{\mu}$

For $k=0, \ldots$

$$
\begin{aligned}
& \mu^{k+1}=\sigma \mu^{k} \\
& \text { solve }(13) \text { for } \delta v^{k} \text { with a relative tolerance of }\left\|\delta v^{k}-\Delta v^{k}\right\|_{H^{1}} \leq \delta\left\|\Delta v^{k}\right\|_{H^{1}} \\
& v^{k+1}=v^{k}+\delta v^{k}
\end{aligned}
$$

Remark 3.2. Algorithm 3.1 is clearly conceptual due to its simplicity. However, using a reliable error estimator and adaptive mesh refinement to satisfy the accuracy requirement $\left\|\delta v^{k}-\Delta v^{k}\right\|_{H^{1}} \leq \delta\left\|\Delta v^{k}\right\|_{H^{1}}$, it can actually be implemented. Details on the implementation of this approach can be found in [16].

In the remainder of the section we show that for suitable choice of $\delta, \sigma$, and $\rho$ this algorithm is well defined and computes iterates that converge to the solution $v(0)$. First we derive a Lipschitz constant for the derivative of $F$, which governs the convergence speed of the exact Newton method.

Lemma 3.3. There is a constant $\omega=\omega\left(\alpha_{\max }, \mu_{\max }\right)$, such that

$$
\left\|\partial_{v} F(v ; \mu)^{-1}\left(\partial_{v} F(v ; \mu)-\partial_{v} F(\hat{v}, \mu)\right)(v-\hat{v})\right\|_{H^{1}} \leq \frac{\omega}{(\alpha+2 \mu) \sqrt{\mu \alpha^{3}}}\|v-\hat{v}\|_{H^{1}}^{2}
$$

holds for all $\left.\alpha \in] 0, \alpha_{\max }\right]$ and $\left.\left.\mu \in\right] 0, \mu_{\max }\right]$.

Proof. First we note that

$$
\partial_{v} F(v ; \mu)=\left[\begin{array}{cc}
I & L \\
L & -u^{\prime}(\lambda)
\end{array}\right] .
$$


By Lemma 2.1 and the Sobolev embedding $H^{1} \subset L^{4}$ for $d \leq 3$ we have

$$
\begin{aligned}
\left\|\left(\partial_{v} F(v ; \mu)-\partial_{v} F(\hat{v}, \mu)\right)(v-\hat{v})\right\|_{L^{2}} & =\left\|\left(u^{\prime}(\hat{\lambda})-u^{\prime}(\lambda)\right)(\lambda-\hat{\lambda})\right\|_{L^{2}} \\
& \leq \frac{1}{\sqrt{\mu \alpha^{3}}}\left\|(\lambda-\hat{\lambda})^{2}\right\|_{L^{2}} \\
& =\frac{1}{\sqrt{\mu \alpha^{3}}}\|\lambda-\hat{\lambda}\|_{L^{4}}^{2} \\
& \leq \frac{1}{\sqrt{\mu \alpha^{3}}}\|\lambda-\hat{\lambda}\|_{H^{1}}^{2}
\end{aligned}
$$

Next we note that $\partial_{v} F(v ; \mu):\left(H_{0}^{1}\right)^{2} \rightarrow\left(H^{-1}\right)^{2}$ satisfies the assumptions of the saddle point Lemma given by [3, Lemma B.1]. In particular, $u^{\prime}(\lambda)$ is a positive semidefinite and bounded Nemyckii operator. We obtain a constant $\gamma$ independent of $\alpha$ and $\mu$, such that

$$
\begin{aligned}
\| \partial_{v} F(v ; \mu)^{-1} & \left(\partial_{v} F(v ; \mu)-\partial_{v} F(\hat{v}, \mu)\right)(v-\hat{v}) \|_{H^{1}} \\
& \leq 4 \max \left(\frac{1}{\gamma}, \frac{1}{\gamma^{2}(\alpha+2 \mu)}\right)\left\|\left(\partial_{v} F(v ; \mu)-\partial_{v} F(\hat{v}, \mu)\right)(v-\hat{v})\right\|_{L^{2}} \\
& \leq \frac{4}{\sqrt{\mu \alpha^{3}}} \max \left(\frac{1}{\gamma}, \frac{1}{\gamma^{2}(\alpha+2 \mu)}\right)\|\lambda-\hat{\lambda}\|_{H^{1}}^{2} .
\end{aligned}
$$

Since $\alpha+2 \mu$ is bounded by $\alpha_{\max }+2 \mu_{\max }$, the claim is verified for $\omega=\max \left(\left(\alpha_{\max }+\right.\right.$ $\left.\left.2 \mu_{\max }\right) / \gamma, \gamma^{-2}\right)$.

Now we are ready to formulate the main result of this section, which takes into account the inexactness of the Newton steps.

Theorem 3.4. With the constant c defined in Theorem 2.3, assume that

$$
\rho=\frac{\sqrt{\alpha^{5}}}{10 \omega}, \quad(1-\sqrt{\sigma}) \leq \frac{\rho}{c}, \quad \sigma \geq \frac{1}{2}, \quad \delta \leq \frac{\sqrt{\sigma}}{4},
$$

and $\left\|v^{0}-v\left(\mu^{0}\right)\right\|_{H^{1}} \leq \rho \sqrt{\mu^{0}}$. Then the iterates defined by Algorithm 3.1 are all well defined and converge linearly towards the limit point v(0). More precisely,

$$
\left\|v^{k}-v\left(\mu^{k}\right)\right\|_{H^{1}} \leq \rho \sqrt{\mu^{k}} \quad \text { and } \quad\left\|v^{k}-v(0)\right\|_{H^{1}} \leq c \sqrt{\mu^{k}}
$$

with some generic constant $c$.

Proof. By induction, assume that (15) holds for some $k$. Using (11) in Theorem 2.3 we derive

$$
\begin{aligned}
\left\|v^{k}-v\left(\mu^{k+1}\right)\right\|_{H^{1}} & \leq\left\|v^{k}-v\left(\mu^{k}\right)\right\|_{H^{1}}+\left\|v\left(\mu^{k}\right)-v\left(\mu^{k+1}\right)\right\|_{H^{1}} \\
& =(\rho+c(1-\sqrt{\sigma})) \sqrt{\mu^{k}} .
\end{aligned}
$$


By the refined Newton-Mysovskii theorem (cf. [6, Thm. 2.3]), one exact Newton step for $\mu=\mu^{k+1}$ yields

$$
\begin{aligned}
\left\|v^{k}+\Delta v^{k}-v\left(\mu^{k+1}\right)\right\|_{H^{1}} & \leq \frac{\omega}{2 \sqrt{\mu^{k+1} \alpha^{5}}}\left\|v^{k}-v\left(\mu^{k+1}\right)\right\|_{H^{1}}^{2} \\
& \leq \frac{\omega}{2 \sqrt{\sigma \mu^{k} \alpha^{5}}}(\rho+c(1-\sqrt{\sigma}))^{2} \mu^{k} \\
& =\frac{\omega}{2 \sigma \sqrt{\alpha^{5}}}(\rho+c(1-\sqrt{\sigma}))^{2} \sqrt{\mu^{k+1}} .
\end{aligned}
$$

The length of the exact Newton correction is bounded by

$$
\begin{aligned}
\left\|\Delta v^{k}\right\|_{H^{1}} & \leq\left\|v\left(\mu^{k+1}\right)-v^{k}-\Delta v^{k}\right\|_{H^{1}}+\left\|v\left(\mu^{k+1}\right)-v^{k}\right\|_{H^{1}} \\
& \leq \frac{\omega}{2 \sigma \sqrt{\alpha^{5}}}(\rho+c(1-\sqrt{\sigma}))^{2} \sqrt{\mu^{k+1}}+(\rho+c(1-\sqrt{\sigma})) \sqrt{\mu^{k}} .
\end{aligned}
$$

Using the assumptions (14) from right to left, we can now estimate the error of the next iterate $v^{k+1}=v^{k}+\delta v^{k}$ obtained by an inexact Newton correction $\delta v^{k}$ as

$$
\begin{aligned}
\| v^{k+1} & -v\left(\mu^{k+1}\right) \|_{H^{1}} \\
& \leq\left[\frac{(1+\delta) \omega}{2 \sigma \sqrt{\alpha^{5}}}(\rho+c(1-\sqrt{\sigma}))^{2}+\frac{\delta}{\sqrt{\sigma}}(\rho+c(1-\sqrt{\sigma}))\right] \sqrt{\mu^{k+1}} \\
& \leq\left[\frac{5 \omega}{8 \sigma \sqrt{\alpha^{5}}}(\rho+c(1-\sqrt{\sigma}))^{2}+\frac{1}{4}(\rho+c(1-\sqrt{\sigma}))\right] \sqrt{\mu^{k+1}} \\
& \leq\left[\frac{5 \omega}{4 \sqrt{\alpha^{5}}}(\rho+c(1-\sqrt{\sigma}))^{2}+\frac{1}{4}(\rho+c(1-\sqrt{\sigma}))\right] \sqrt{\mu^{k+1}} \\
& \leq\left[\frac{5 \omega}{4 \sqrt{\alpha^{5}}}(2 \rho)^{2}+\frac{1}{4}(2 \rho)\right] \sqrt{\mu^{k+1}} \\
& =\left[\frac{5 \omega}{\sqrt{\alpha^{5}}} \rho+\frac{1}{2}\right] \rho \sqrt{\mu^{k+1}} \\
& =\rho \sqrt{\mu^{k+1}},
\end{aligned}
$$

which completes the induction.

Remark 3.5. Exploiting strong strict complementarity of the solution, local superlinear convergence of a similar short step pathfollowing method can be shown $[17]$.

\section{Finite element discretization}

The advantage of eliminating the control pointwise is apparent when it comes to discretize the variables. Remember that Algorithm 3.1 requires the solution of (13) up to a relative (discretization) error of $\left\|\delta v^{k}-\Delta v^{k}\right\|_{H^{1}} \leq \delta\left\|\Delta v^{k}\right\|_{H^{1}}$. 
We consider finite element discretizations on a sequence of uniformly shaperegular and quasi-uniform triangulations $\mathcal{T}_{h}$ of $\Omega$ with maximal element size $h$, thus we assume that there exists a constant $c$, such that $h_{\min } \geq c h$. On $\mathcal{T}_{h}$ we define for $p=1,2$ the ansatz space $V_{h}^{p}=\left\{\phi \in\left(H_{0}^{1}(\Omega)\right)^{2}\left|\forall T \in \mathcal{T}_{h}: \phi\right|_{T} \in \mathbb{P}_{p}^{2}\right\}$ of piecewise linear and piecewise quadratic functions, respectively. With $I_{h}$ : $C^{0}(\Omega) \rightarrow V_{h}^{p}$ we denote the Lagrange interpolation operator with interpolation points at the vertices of $\mathcal{T}_{h}$ for $p=1$, and at vertices and edge midpoints of $\mathcal{T}_{h}$ for $p=2$, respectively.

Since the accuracy of the numerical integration used for assembling $\langle u, \phi\rangle$ turns out to be crucial, it is considered separately from the finite element discretization. We interpret the numerical integration as exact integration of a projection $P_{h} u$ of $u$. The discrete approximation $F_{h}: V_{h}^{p} \rightarrow\left(V_{h}^{p}\right)^{*}$ is then defined by

$\left\langle F_{h}\left(v_{h}\right),\left(\phi_{1}, \phi_{2}\right)\right\rangle=\left\langle y_{h}-y_{d}+L \lambda_{h}, \phi_{1}\right\rangle+\left\langle L y_{h}-P_{h} u\left(\lambda_{h}\right), \phi_{2}\right\rangle \quad \forall\left(\phi_{1}, \phi_{2}\right) \in V_{h}^{p}$.

With $S_{h}: H^{-1} \rightarrow V_{h}^{p}, z \mapsto \zeta$, we denote the solution operator of the discretized problem

$$
\langle L \zeta-z, \phi\rangle=0 \quad \forall \phi \in V_{h}^{p}
$$

with exact integration. For a triangulation $\mathcal{T}$ we denote by

$$
\|v\|_{H^{2}, \mathcal{T}}:=\|v\|_{H^{1}(\Omega)}+\sum_{T \in \mathcal{T}}\|v\|_{H^{2}(T)}
$$

the piecewise $H^{2}$-norm and by $H_{\mathcal{T}}^{2}$ the corresponding Sobolev space.

Lemma 4.1. Assume that $y_{d} \in H^{1}$. Then there exists a constant $c<\infty$ independent of $\mu$, such that the central path solutions $(y, \lambda)$ satisfy the following regularity conditions:

$$
\|y\|_{H^{2}} \leq c, \quad\|\lambda\|_{H^{3}} \leq c .
$$

Moreover, for $\lambda \in W^{1,4}(\Omega) \cap H_{\mathcal{T}}^{2}(\Omega)$ the following regularity estimates hold for $u(\lambda ; \mu)$ :

$$
\begin{aligned}
& \|u(\lambda ; \mu)\|_{L_{2}} \leq c, \quad\|u(\lambda ; \mu)\|_{H^{1}} \leq c\|\lambda\|_{H^{1}} \\
& \|u(\lambda ; \mu)\|_{H^{2}, \mathcal{T}} \leq c\|\lambda\|_{H^{2}, \mathcal{T}}+\frac{c}{\sqrt{\mu}}\|\lambda\|_{W^{1,4}}^{2} .
\end{aligned}
$$

Proof. We denote by $c$ a generic constant which is independent of $\mu$. Since $|u| \leq 1$ by construction, $\|u(\lambda)\|_{L_{2}} \leq \sqrt{|\Omega|}$ is immediately clear. By standard regularity results for elliptic PDEs (see e.g. [8]) we have $\|y\|_{H^{2}} \leq c\|u\|_{L^{2}} \leq$ $c$ and $\|\lambda\|_{H^{3}} \leq c\left\|y-y_{d}\right\|_{H^{1}} \leq c$. Lemma 2.1 and $\nabla u=u^{\prime}(\lambda) \nabla \lambda$ imply $\|u\|_{H^{1}} \leq \frac{1}{\alpha}\|\lambda\|_{H^{1}} \leq c$. Concerning the $H^{2}$-estimate for $u$ we compute $\nabla^{2} u=$ $u^{\prime \prime}(\lambda)(\nabla \lambda, \nabla \lambda)+u^{\prime}(\lambda) \nabla^{2} \lambda$ and conclude

$$
\|u\|_{H^{2}, \mathcal{T}} \leq c\left\|u^{\prime \prime}\right\|_{\infty}\|\lambda\|_{W^{1,4}}^{2}+\left\|u^{\prime}\right\|_{\infty}\|\lambda\|_{H^{2}, \mathcal{T}},
$$

which yields the assertion. 
In the following subsection, we derive discretization error estimates based on certain accuracy assumptions imposed on $P_{h}$, whereas the next subsection is devoted to the question how to realize an integration scheme that satisfies these assumptions.

\subsection{Error estimates}

First we collect some immediate results of standard finite element theory.

Lemma 4.2. The following estimates hold for $p=1,2$ :

$$
\begin{aligned}
\|S z\|_{H^{m}} & \leq c\|z\|_{H^{m-2}} \quad \text { for } m=1,2,3 \\
\left\|\left(S_{h}-S\right) z\right\|_{H^{1}} & \leq c h^{p}\|z\|_{H^{p-1}} \\
\left\|\left(S_{h}-S\right) z\right\|_{L^{2}} & \leq c h^{p+1}\|z\|_{H^{p-1}} \\
\left\|\left(S_{h} S_{h}-S S\right) z\right\|_{L^{2}} & \leq c h^{p+1}\|z\|_{H^{1}} \\
\left\|S_{h} z\right\|_{H^{2}, \mathcal{T}} & \leq c\|z\|_{H^{1}}
\end{aligned}
$$

Proof. The regularity result (16) holds for sufficiently smooth $\partial \Omega$ (see e.g. [8]). For (17) and (18) we refer to [5, Thms. 3.2.2 and 3.2.5]. As for (19) we estimate

$$
\begin{aligned}
\left\|\left(S_{h} S_{h}-S S\right) z\right\|_{L^{2}} & \leq\left\|S_{h}\left(S_{h}-S\right) z\right\|_{L^{2}}+\left\|\left(S_{h}-S\right) S z\right\|_{L^{2}} \\
& \leq c\left\|\left(S_{h}-S\right) z\right\|_{L^{2}}+c h^{p+1}\|S z\|_{H^{p-1}} \\
& \leq c h^{p+1}\|z\|_{H^{p-1}}+c h^{p+1}\|z\|_{L^{2}} \\
& \leq c h^{p+1}\|z\|_{H^{1}} .
\end{aligned}
$$

Concerning (20), we only need to consider the case $p=2$, and we first notice that $S z \in H^{3}$. Thus the Lagrange interpolate $I_{h} S z$ of $S z$ corresponding to the quadratic finite elements satisfies the error estimates (cf. [5, Thm. 3.1.6])

$$
\begin{aligned}
\left\|I_{h} S z-S z\right\|_{H^{2}, \mathcal{T}} & \leq c h\|S z\|_{H^{3}} \leq c h\|z\|_{H^{1}} \\
\left\|I_{h} S z-S z\right\|_{H^{1}} & \leq c h^{2}\|S z\|_{H^{3}} \leq h^{2} c\|z\|_{H^{1}},
\end{aligned}
$$

and by (17) we obtain

$$
\left\|I_{h} S z-S_{h} z\right\|_{H^{1}} \leq\left\|I_{h} S z-S z\right\|_{H^{1}}+\left\|S_{h} z-S z\right\|_{H^{1}} \leq c h^{2}\|z\|_{H^{1}} .
$$

Now we may use an inverse inequality $\left\|v_{h}\right\|_{H^{2}, \mathcal{T}} \leq \frac{c}{h}\left\|v_{h}\right\|_{H^{1}} \forall v_{h} \in V_{h}^{2}$ (cf. [5, Thm. 3.2.6]) to obtain

$$
\begin{aligned}
\left\|S_{h} z\right\|_{H^{2}, \mathcal{T}} & \leq\left\|I_{h} S z-S_{h} z\right\|_{H^{2}, \mathcal{T}}+\left\|I_{h} S z-S z\right\|_{H^{2}, \mathcal{T}}+\|S z\|_{H^{2}, \mathcal{T}} \\
& \leq c(1+h)\|z\|_{H^{1}} .
\end{aligned}
$$

Lemma 4.3. Consider a quasi-uniform family of triangulations $\mathcal{T}_{h}$ of $\Omega$, let $p=1,2, z \in H^{p+1}(\Omega), z_{h} \in V_{h}^{p}(\Omega)$ and assume

$$
\left\|z-z_{h}\right\|_{H^{1}} \leq c h^{p}\|z\|_{H^{p+1}} .
$$


Then the following discrete Sobolev inequalitiy holds with $c$ independent of $h$ :

$$
\left\|z_{h}\right\|_{W^{1,4}} \leq c\|z\|_{H^{p+1}}
$$

Proof. We use the triangle inequality to obtain

$$
\left\|z_{h}\right\|_{W^{1,4}(\Omega)} \leq\|z\|_{W^{1,4}(\Omega)}+\left\|I_{h} z-z\right\|_{W^{1,4}(\Omega)}+\left\|z_{h}-I_{h} z\right\|_{W^{1,4}(\Omega)} .
$$

Thus we may estimate on each triangle (cf. [5, Thm. 3.1.6]):

$$
\left\|I_{h} z-z\right\|_{W^{1,4}(T)} \leq c h^{p+d(1 / 4-1 / 2)}\|z\|_{H^{p+1}(T)} .
$$

Moreover, an inverse inequality (cf. [5, Thm. 3.2.6]) applied to the grid function $z_{h}-I_{h} z$ yields

$$
\begin{aligned}
\left\|z_{h}-I_{h} z\right\|_{W^{1,4}(T)} & \leq c h^{d(1 / 4-1 / 2)}\left\|z_{h}-I_{h} z\right\|_{H^{1}(T)} \\
& \leq c h^{-d / 4}\left(\left\|z_{h}-z\right\|_{H^{1}(T)}+\left\|z-I_{h} z\right\|_{H^{1}(T)}\right),
\end{aligned}
$$

where $d$ is the spatial dimension. Using the estimate

$$
\left\|z-I_{h} z\right\|_{H^{1}(T)} \leq h^{p}\left\|z-I_{h} z\right\|_{H^{p+1}(T)}
$$

we obtain together:

$$
\left\|z_{h}\right\|_{W^{1,4}(T)} \leq\|z\|_{W^{1,4}(T)}+c h^{p-d / 4}\|z\|_{H^{p+1}(T)}+c h^{-d / 4}\left\|z_{h}-z\right\|_{H^{1}(T)}
$$

To sum up over all triangles we use the following relations in $l_{q}$-spaces:

$$
\left\|v_{1}+v_{2}\right\|_{l_{4}} \leq\left\|v_{1}\right\|_{l_{4}}+\left\|v_{2}\right\|_{l_{4}} \leq\left\|v_{1}\right\|_{l_{4}}+\left\|v_{2}\right\|_{l_{2}}
$$

insert $v_{1}:=\left(\|z\|_{W^{1,4}(T)}\right)_{T \in \mathcal{T}_{h}}$ and $v_{2}:=\left(c h^{p-d / 4}\|z\|_{H^{p+1}(T)}+c h^{-d / 4} \| z_{h}-\right.$ $\left.z \|_{H^{1}(T)}\right)_{T \in \mathcal{T}_{h}}$ and obtain (since $p-d / 4>0$ ):

$$
\begin{aligned}
\left\|z_{h}\right\|_{W^{1,4}(\Omega)} & \leq\|z\|_{W^{1,4}(\Omega)}+c h^{p-d / 4}\|z\|_{H^{p+1}(\Omega)}+c h^{-d / 4}\left\|z_{h}-z\right\|_{H^{1}(\Omega)} \\
& \leq\|z\|_{W^{1,4}(\Omega)}+c h^{p-d / 4}\|z\|_{H^{p+1}(\Omega)}+c h^{p-d / 4}\|z\|_{H^{p+1}(\Omega)} \\
& \leq\|z\|_{W^{1,4}(\Omega)}+c\|z\|_{H^{p+1}(\Omega)} .
\end{aligned}
$$

Here we used the estimate

$$
h_{\min }^{-d / 4}\left\|z_{h}-z\right\|_{H^{1}(\Omega)} \leq c h^{-d / 4}\left\|z_{h}-z\right\|_{H^{1}(\Omega)}
$$

and thus quasi-uniformity of the triangulation. Finally, the Sobolev embedding $H^{p+1}(\Omega) \hookrightarrow W^{1,4}(\Omega)$ yields:

$$
\left\|z_{h}\right\|_{W^{1,4}} \leq c\|z\|_{H^{p+1}}
$$


Lemma 4.4. Let $c$ denote some generic constant independent of $\mu, u, h$, and $v$. If the linear projector $P_{h}$ satisfies

$$
\left\|P_{h} u-u\right\|_{H^{-1}} \leq c h^{p} \sqrt{\mu}\|u\|_{H^{2}, \mathcal{T}},
$$

then there is a discrete central path solution $v_{h} \in V_{h}^{p}$ with associated control $u_{h}=u\left(\lambda_{h}\right)$, such that for both linear $(p=1)$ and quadratic $(p=2)$ finite elements the error estimates

$$
\begin{aligned}
& \left\|v_{h}-v(\mu)\right\|_{H^{1}} \leq c h^{p} \\
& \left\|u_{h}-u(\mu)\right\|_{L^{2}} \leq c h^{p}
\end{aligned}
$$

hold.

Proof. (i) Existence of $v_{h}$. The set $M=\left\{u \in L^{2}:|u| \leq 1\right.$ a.e. $\}$ is nonempty, closed, convex, and bounded, and the mapping

$$
T: L^{2} \rightarrow L^{2}, u \mapsto u\left(\lambda_{h}\left(y_{h}\left(P_{h} u\right)-y_{d}\right)\right)=u\left(-S_{h}\left(S_{h} P_{h} u-y_{d}\right)\right)
$$

is a compact operator that maps $M$ into itself. By the Schauder fixed-point theorem (cf. [23, Theorem 2.A]) $T$ has a fixed point $u_{h}=u\left(-S_{h}\left(S_{h} P_{h} u-y_{d}\right)\right)$ and corresponding finite element solutions $v_{h}=\left(y_{h}, \lambda_{h}\right)$, such that $F_{h}\left(v_{h}\right)=0$. (ii) Inexact integration of $u_{h}$. Define $\lambda_{h}:=-S_{h}\left(y_{h}-y_{d}\right)$ and $\tilde{\lambda}:=-S\left(y_{h}-y_{d}\right)$. Since $y_{h}-y_{d} \in H^{1}$, it follows that $\tilde{\lambda} \in H^{p+1}$ and by Lemma 4.3:

$$
\left\|\lambda_{h}-\tilde{\lambda}\right\|_{H^{1}} \leq c h^{p}\|\tilde{\lambda}\|_{H^{p+1}} \Rightarrow\left\|\lambda_{h}\right\|_{W^{1,4}} \leq c, \quad\left\|\lambda_{h}\right\|_{H^{2}, \mathcal{T}} \leq c
$$

and thus by Lemma 4.1

Assumption (23) yields

$$
\left\|u_{h}\right\|_{H^{2}, \mathcal{T}} \leq c \frac{1}{\sqrt{\mu}}
$$

$$
\begin{aligned}
\left\|S_{h}\left(P_{h} u_{h}-u_{h}\right)\right\|_{H^{1}} & \leq c\left\|P_{h} u_{h}-u_{h}\right\|_{H^{-1}} \\
& \leq c h^{p} \sqrt{\mu}\left\|u_{h}\right\|_{H^{2}, \mathcal{T}} \leq c h^{p} .
\end{aligned}
$$

(iii) $L^{2}$ error estimates for $u_{h}$. First we note that

$$
\lambda(u)=\alpha u-\frac{\mu}{u+1}+\frac{\mu}{1-u}
$$

obtained from (8) is monotonically increasing with $\lambda^{\prime}(u) \geq \alpha+\mu$, and holds for both the exact central path solution as well as for any discrete solution $\lambda_{h}=-S_{h}\left(S_{h} P_{h} u-y_{d}\right)$. We adapt the proof given in [10] to the present setting and estimate

$$
\begin{aligned}
(\alpha+\mu)\left\|u-u_{h}\right\|_{L^{2}}^{2} \leq & \left\langle\lambda(u)-\lambda\left(u_{h}\right), u-u_{h}\right\rangle \\
= & \left\langle-S\left(S u-y_{d}\right)+S_{h}\left(S_{h} P_{h} u_{h}-y_{d}\right), u-u_{h}\right\rangle \\
= & \left\langle-S S u+S y_{d}+S_{h} S_{h} P_{h} u_{h}-S_{h} y_{d}, u-u_{h}\right\rangle \\
= & \left\langle\left(S_{h} S_{h}-S S\right) u, u-u_{h}\right\rangle+\underbrace{\left\langle S_{h} S_{h}\left(u_{h}-u\right), u-u_{h}\right\rangle}_{\leq 0} \\
& +\left\langle S_{h} S_{h}\left(P_{h} u_{h}-u_{h}\right), u-u_{h}\right\rangle+\left\langle\left(S-S_{h}\right) y_{d}, u-u_{h}\right\rangle .
\end{aligned}
$$


Dividing by $\left\|u-u_{h}\right\|_{L^{2}}$ we obtain by (24)

$$
\begin{aligned}
(\alpha+\mu)\left\|u-u_{h}\right\|_{L^{2}} & \left.\leq\left\|\left(S_{h} S_{h}-S S\right) u\right\|_{L^{2}}+\| S_{h} S_{h}\left(P_{h}-I\right) u_{h}\right)\left\|_{L^{2}}+\right\|\left(S-S_{h}\right) y_{d} \|_{L^{2}} \\
& \leq c h^{p+1}\|u\|_{H^{1}}+c h^{p} \sqrt{\mu}\left\|u_{h}\right\|_{H^{2}, \mathcal{T}}+c h^{p+1}\left\|y_{d}\right\|_{H^{1}} \\
& \leq c h^{p} .
\end{aligned}
$$

(iv) $H^{1}$ error estimates for $y_{h}$ and $\lambda_{h}$. Equipped with the estimate $\left\|u-u_{h}\right\|_{L^{2}} \leq$ $c h^{p}$ we obtain by standard error estimates for finite element solutions and (24)

$$
\begin{aligned}
\left\|y-y_{h}\right\|_{H^{1}} & \leq\left\|S u-S u_{h}\right\|_{H^{1}}+\left\|S u_{h}-S_{h} u_{h}\right\|_{H^{1}}+\left\|S_{h} u_{h}-S_{h} P_{h} u_{h}\right\|_{H^{1}} \\
& \leq c\left\|u-u_{h}\right\|_{L^{2}}+c h^{p}\left\|u_{h}\right\|_{H^{p-1}}+c h^{p} \sqrt{\mu}\left\|u_{h}\right\|_{H^{2}, \mathcal{T}} \\
& \leq c h^{p}
\end{aligned}
$$

and

$$
\begin{aligned}
\left\|\lambda-\lambda_{h}\right\|_{H^{1}} & \leq\left\|S\left(y-y_{h}\right)\right\|_{H^{1}}+\left\|\left(S_{h}-S\right) y_{h}\right\|_{H^{1}} \\
& \leq c h^{p}+c h^{p}\left\|y_{h}\right\|_{H^{p-1}} \\
& \leq c h^{p} .
\end{aligned}
$$

Corollary 4.5. If (23) holds, Algorithm 3.1 computes discrete central path solutions $v_{h} \in V_{h}^{p}$ with associated control $u_{h}$ with distance to the solution $v(0)$ of (1) bounded by

$$
\begin{aligned}
\left\|v_{h}-v(0)\right\|_{H^{1}} & \leq c h^{p} \\
\left\|u_{h}-u(0)\right\|_{L^{2}} & \leq c h^{p} .
\end{aligned}
$$

Proof. For $\mu>0$, Theorem 3.4 requires discrete solutions $v_{h}$ with $\left\|v_{h}-v(\mu)\right\|_{H^{1}} \leq$ $\rho \sqrt{\mu}$, which is satisfied for

$$
c h^{p} \leq \rho \sqrt{\mu}
$$

by Lemma 4.4. Using the coarsest possible discretization, i.e. choosing $h(\mu)$ or equivalently $\mu(h)$ such that equality holds in (25), we obtain

$$
\left\|v_{h}-v(0)\right\|_{H^{1}} \leq\left\|v_{h}-v(\mu)\right\|_{H^{1}}+\|v(\mu)-v(0)\|_{H^{1}} \leq c h^{p}
$$

by Theorem 2.3 .

Remark 4.6. Higher order results $\left\|u_{h}-u(0)\right\|_{L^{2}} \leq c h^{p+1-\varepsilon}$ can be obtained by using more accurate numerical integration and superconvergence properties of the central path (cf. $[16,17])$, if a strong strict complementarity assumption holds for the solution $v(0)$. 


\subsection{Numerical integration schemes}

We will now analyse a simple possibility to construct an interpolation operator $P_{h}$ that satisfies (23). Since we only have regularity in the norm $\|\cdot\|_{H^{2}, \mathcal{T}}$, we define $P_{h}=P_{h, \tilde{h}}$ as a piecewise linear interpolation on sub-triangles with size $\tilde{h} \leq h$, that are aligned to the finite-element grid. By standard interpolation theory we obtain the error estimate

$$
\left\|P_{h} u-u\right\|_{L^{2}} \leq c \tilde{h}^{2}\|u\|_{H^{2}, \mathcal{T}}
$$

thus we have to choose

$$
\tilde{h}^{2}:=c h^{p} \sqrt{\mu}
$$

By (25) we know that $h^{p}=c \rho \sqrt{\mu}$ holds during Algorithm 3.1. Inserting this into (26) yields

$$
\tilde{h^{2}}:=c h^{2 p},
$$

and hence

$$
\tilde{h}=c h^{p} \text {. }
$$

Thus, in the case of linear finite elements we may use a fixed subdivision of the finite element grid to interpolate $u_{h}$, but in the case of quadratic finite elements we have to introduce finer and finer subgrids for assembling $u_{h}$ sufficiently accurate.

Taking the considerations above into account, the computational effort for evaluating the integrals during the assembly phase up to the required accuracy is a significant part of the overall computational work. In contrast, control discretization approaches can work with fixed, standard quadrature rules, but require a significantly finer mesh in order to achieve the same accuracy. Therefore, their computational complexity for assembly is comparable to the control reduced method, but their effort for solution of the resulting large systems is considerably greater. Furthermore, their memory requirement for storing fine meshes and the associated data is much greater.

Remark 4.7. In actual computation with quadratic finite elements one will choose the accuracy of the integration adaptively. If the boundary $\partial \Omega_{A}$ of the active set $\Omega_{A}$ of $u$ is not too complex, the additional computational effort for such an adaptive integration will be bounded by a fixed factor, since sharp bends in $u_{h}$ are to be expected in the vicinity of $\partial \Omega_{A}$ only. Moreover, the contribution of this region to the overall error will be small, due to its shrinking size.

\section{Numerical examples}

This section is devoted to demonstrate the method at some illustrative examples. 

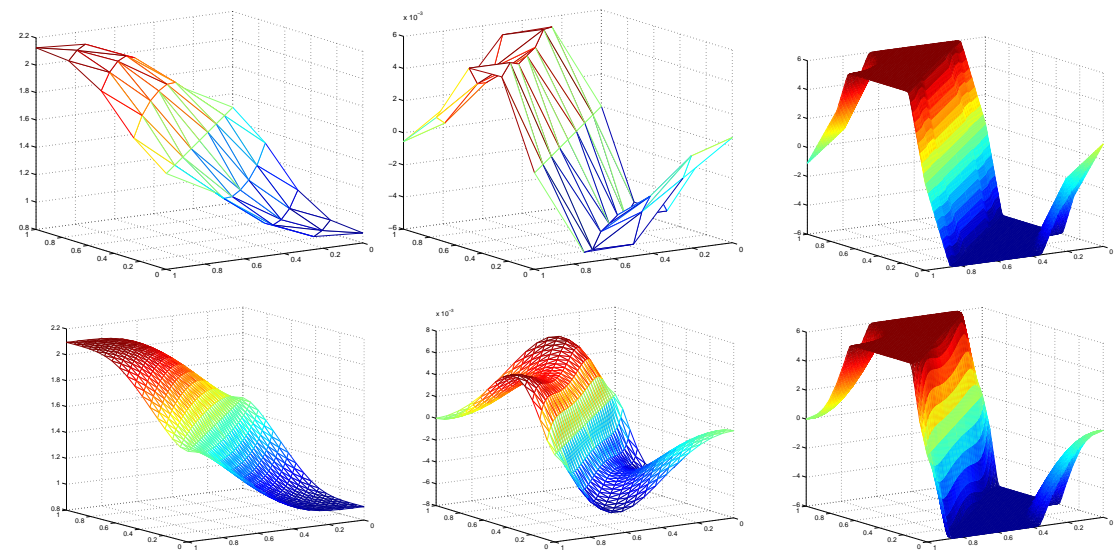

Figure 1: Discrete solution at $\mu=2^{-18}$. From left to right: state $y$, multiplier $\lambda$, and control $u$. Top row: $h=1 / 4$. Bottom row: $h=1 / 16$. In order to display the accuracy gain, the control is interpolated on a significantly finer grid than $y$ and $\lambda$ were computed on.

Example 1. As a completely artificial example we choose

$$
\begin{gathered}
\min _{y \in H^{1}, u \in L^{2}} \frac{1}{2}\left\|y-y_{d}\right\|_{L^{2}}^{2}+\frac{\alpha}{2}\|u\|_{L^{2}}^{2} \\
\text { subject to } \quad-\Delta y=u \quad \text { in } \Omega, \quad \partial_{n} y=0 \quad \text { on } \partial \Omega, \quad-6 \leq u \leq 6
\end{gathered}
$$

with $\Omega=[0,1]^{2}, \alpha=5 e-4$, and

$$
y_{d}=\left\{\begin{array}{ll}
1, & x_{1}+x_{2}<1 \\
2, & \text { otherwise }
\end{array} .\right.
$$

In order to examine the numerical convergence properties with respect to the mesh size $h$ we employ uniform criss-cross triangulations with $h=2^{-n}$. Note that the assumption $\partial \Omega \in C^{3}$ is violated, such that the state equation is not $H^{3}$-regular. It is, however, $H^{2}$-regular due to the convexity of $\Omega$, which is all that is needed for proving the convegence rates for linear elements $(p=1)$. For quadratic elements, the numerically observed convergence rates do almost match the theoretical results obtained for $H^{3}$ regular problems. This can be attributed to the fact that $H^{3}$ regularity is lost only in the vicinity of the corner points of $\Omega$, such that the impact on the overall error is almost negligible.

Discrete central path solutions for different mesh sizes are shown in Figure 1, with a zoom into the control given in Figure 2. The structure of the control and in particular shape and location of the developing kinks are surprisingly well represented already on very coarse meshes.

This translates into the maximal order of convergence that can be expected for linear and quadratic finite elements, respectively. Discretization errors for 
state $y$ and multiplier $\lambda$, both in $\|\cdot\|_{H^{1}}$ and $\|\cdot\|_{L^{2}}$, are given in Figure 3 . Note that the quadratic and cubic convergence order of $\lambda$ in $L^{2}$ for linear and quadratic elements, respectively, translates directly into the same order of convergence for $u$ in $L^{2}$.
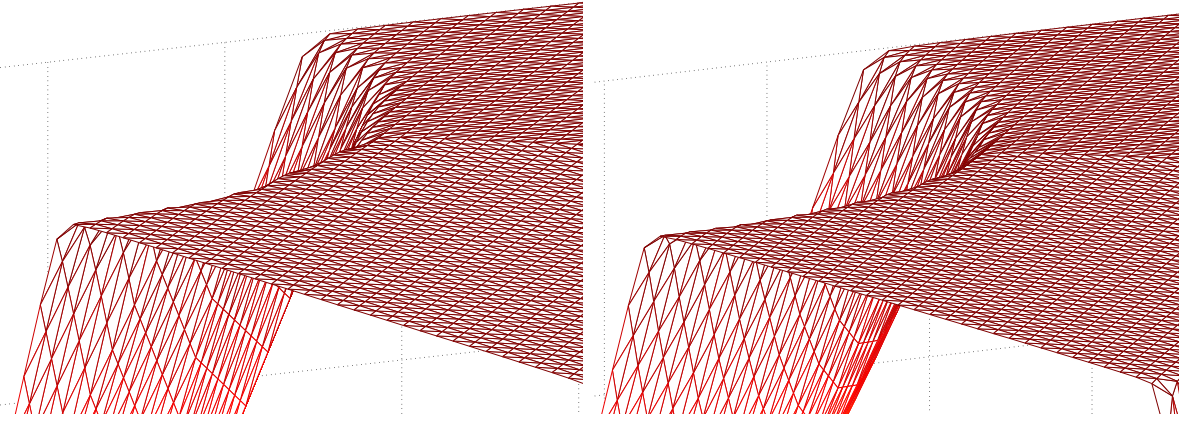

Figure 2: Zoom of control for $\mu=2^{-18}$. Left: $h=1 / 4$. Right: $h=1 / 16$.
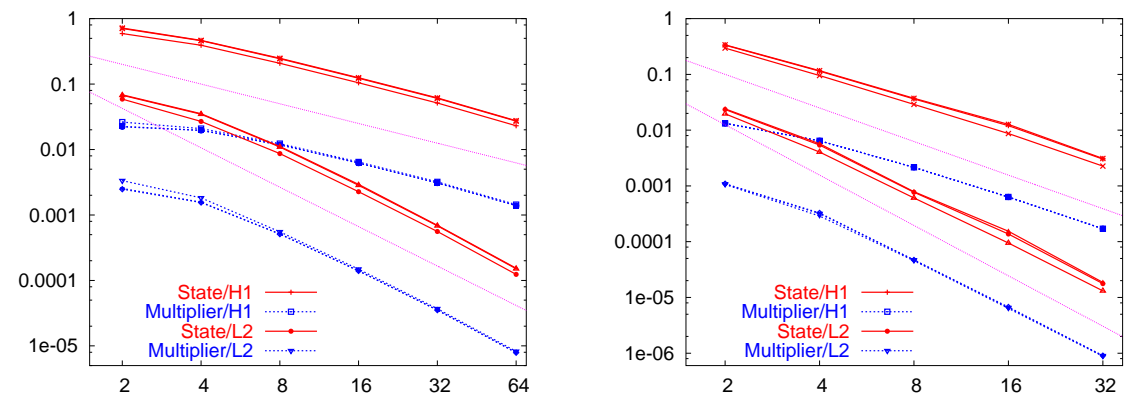

Figure 3: Convergence rates for linear (left) and quadratic (right) finite elements at different values of $\mu=2^{-8}, 2^{-13}, 2^{-15}$.

Example 2. This problem taken from [22] is a drastically simplified benchmark problem for applicator development in regional hyperthermia treatment, a cancer therapy that aims at heating deeply seated tumors by microwave radiation in order to make it more susceptible to an accompanying radio or chemo therapy [7]. The governing PDE is the stationary bio-heat-transfer equation [13]

$$
\begin{aligned}
-\nabla(\kappa \nabla y)+(y-37) w & =\sigma u & & \text { in } \Omega \\
\partial_{n} y & =0 & & \text { on } \partial \Omega
\end{aligned}
$$

for the temperature $y$ on the relevant part $\Omega$ of the human body. Our benchmark problem is based on a problem considered in [2, Section 4], where a detailed description of the material parameters can be found. 

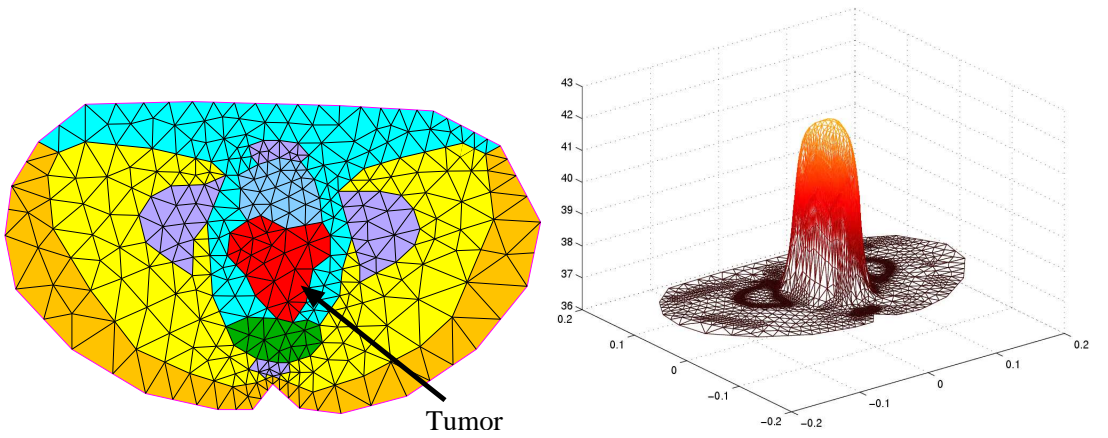

Figure 4: Cross section $\Omega$ of the pelvic region with different tissue types (left) and optimal state (right).

The control $u$, assumed to be freely adjustable within the bounds $0 \leq u \leq$ $u_{\max }$, is the energy absorption of the tissue and is directly related to the amplitude of the time harmonic electric field generated by the microwave generator. We set $u_{\max }:=10^{6} \mathrm{~V}^{2} / \mathrm{m}^{2}$. The thermal effect of perfusion $w$ with arterial blood of $37^{\circ} \mathrm{C}$ from different regions of the body is accounted for by the Helmholtz term. We aim at a therapeutical temperature

$$
y_{d}= \begin{cases}45 & \text { in } \Omega_{t} \\ 37 & \text { in } \Omega \backslash \Omega_{t}\end{cases}
$$

that affects only the tumor tissue $\Omega_{t} \subset \Omega$ (see Fig. 4). For this example, the regularization parameter $\alpha$ has been set to $10^{-12}$.

As can be expected, the optimal control just deposits almost all the energy into the tumor region and almost nothing outside. The very small value of $\alpha$ leads to a very thin band of steep increase in the control, which is, however, not aligned to the coarse grid.

The intent of this example is not to numerically verify the theoretical convergence rates for $h \rightarrow 0$, but to illustrate the efficiency of the method for rather coarse meshes. In particular, here we are not interested in the loss of even $H^{2}$-regularity due to the nonconvex polygonal domain $\Omega$.

The problem has been solved numerically by a primal-dual function space oriented interior point method as described in [21, 22]. The discretized control needs to represent the 'discontinuity' of the solution with sufficient accuracy, such that massive grid refinement occurs along the boundaries of the active sets (see Fig. 5, left). The finest computational grid contained about 40000 triangles, most of them concentrated along the control 'discontinuity'.

We also solved this problem with the control reduced primal interior point method, however on a much coarser grid with about 2500 triangles. Then the control was computed via (8) on a uniformly refined grid (see Fig. 5, right), which yielded a comparable result with much less computational effort. 

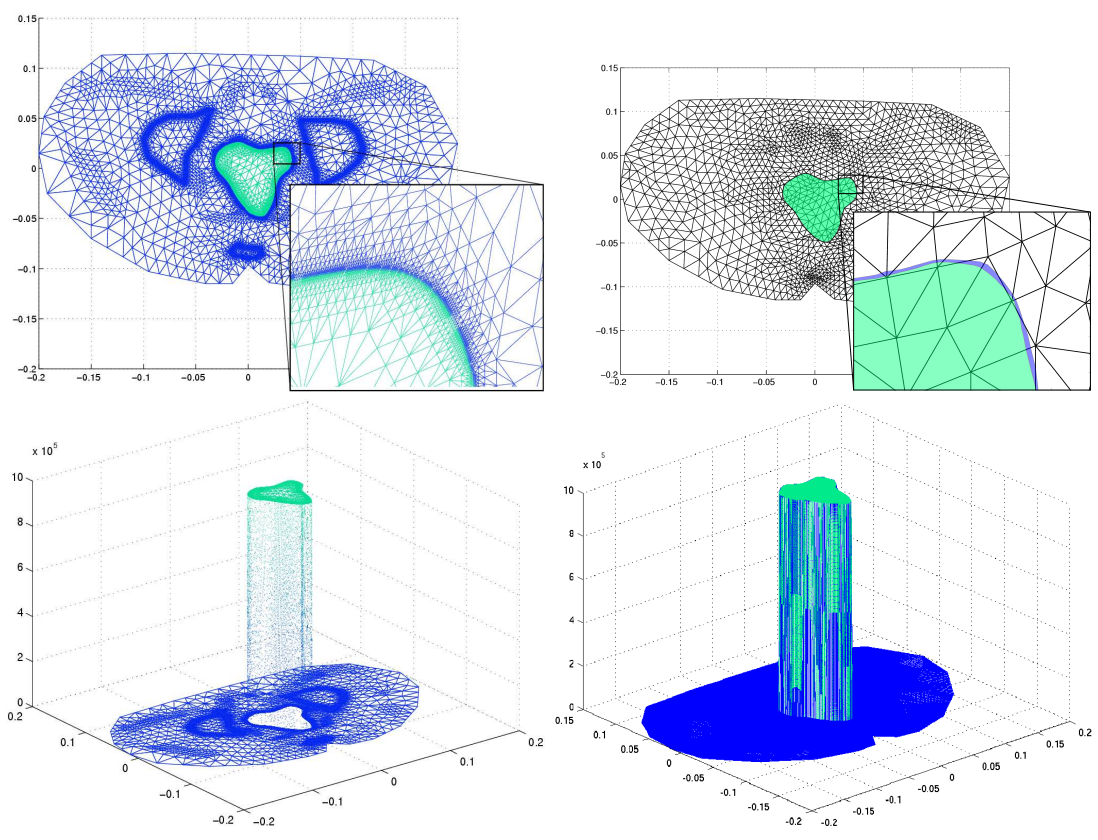

Figure 5: Mesh refinement and discrete control for the primal dual approach with piecewise constant control discretization (left) and for the control reduced primal approach (right) computed on a coarse grid. 


\section{Conclusion}

A novel discretization scheme for primal interior point methods applied to PDE constrained optimization problems has been presented. Pointwise elimination of the control, which is the least regular variable, enables high accuracy with comparatively coarse meshes.

\section{References}

[1] N. Arada, E. Casas, and F. Tröltzsch. Error estimates for the numerical approximation of a semilinear elliptic control problem. Comput. Optim. Appl., 23:201-229, 2002.

[2] F.A. Bornemann. An adaptive multilevel approach to parabolic equations III. 2D error estimation and multilevel preconditioning. IMPACT Comput. Sci. Engrg., 4:1-45, 1992.

[3] D. Braess and C. Blömer. A multigrid method for a parameter dependent problem in solid mechanics. Numer. Math., 57:747-761, 1990.

[4] E. Casas and F. Tröltzsch. Error estimates for linear-quadratic elliptic control problems. In V. Barbu et al., editor, Analysis and Optimization of Differential Systems, pages 89-100. Kluwer, 2003.

[5] P.G. Ciarlet. The finite element method for elliptic problems. NorthHolland, 1987.

[6] P. Deuflhard. Newton Methods for Nonlinear Problems. Affine Invariance and Adaptive Algorithms, volume 35 of Computational Mathematics. Springer, 2004.

[7] P. Deuflhard, M. Seebaß, D. Stalling, R. Beck, and H.-C. Hege. Hyperthermia Treatment Planning in Clinical Cancer Therapy: Modelling, Simulation and Visualization. In A. Sydow, editor, Proc. of the 15th IMACS World Congress 1997 on Scientific Computation: Modelling and Applied Mathematics, volume 3, pages 9-17. Wissenschaft und Technik Verlag, 1997.

[8] D. Gilbarg and N.S. Trudinger. Elliptic Partial Differential Equations of Second Order. Springer, 1977.

[9] M. Hintermüller, K. Ito, and K Kunisch. The primal-dual active set strategy as a semi-smooth Newton method. SIAM J. Optim., 13:865-888, 2002.

[10] M. Hinze. A variational discretization concept in control constrained optimization: the linear-quadratic case. Comput. Optim. Appl., 30:45-63, 2005.

[11] B. Kummer. Generalized Newton and NCP-methods: convergence, regularity and actions. Discussiones Mathematicae - Differential Inclusions, 20(2):209-244, 2000. 
[12] C. Meyer and A. Rösch. Superconvergence properties of optimal control problems. SIAM J. Control Optim., 43(3):970-985, 2004.

[13] H.H. Pennes. Analysis of tissue and arterial blood temperatures in the resting human forearm. J. Appl. Phys., 1:93-122, 1948.

[14] U. Prüfert, F. Tröltzsch, and M. Weiser. The convergence of an interior point method for an elliptic control problem with mixed control-state constraints. Comput. Optim. Appl., to appear.

[15] A. Rösch. Error estimates for linear-quadratic control problems with control constraints. Optim. Meth. Softw., 21(1):121-134, 2006.

[16] A. Schiela. The Control Reduced Interior Point Method. doctoral thesis, Freie Universität Berlin, Dept. Math. and Comp. Sci., 2006.

[17] A. Schiela and M. Weiser. Superlinear convergence of the control reduced interior point method for PDE constrained optimization. Comput. Optim. Appl., to appear.

[18] M. Ulbrich. Nonsmooth Newton-like Methods for Variational Inequalities and Constrained Optimization Problems in Function Spaces. Habilitation thesis, Fakultät für Mathematik, Technische Universität München, 2002.

[19] M. Ulbrich and S. Ulbrich. Primal-dual interior point methods for PDEconstrained optimization. Technical report, TU Darmstadt, 2006.

[20] M. Weiser. Interior point methods in function space. SIAM J. Control Optim., 44(5):1766-1786, 2005.

[21] M. Weiser and P. Deuflhard. Inexact central path following algorithms for optimal control problems. SIAM J. Control Optim., to appear.

[22] M. Weiser and A. Schiela. Function space interior point methods for PDE constrained optimization. PAMM, 4(1):43-46, 2004.

[23] E. Zeidler. Nonlinear Functional Analysis and its Applications, volume I. Springer, New York, 1986. 